Meta

Journal des traducteurs

Translators' Journal

\title{
Grammar and Translation: The Noun + Noun Conundrum
}

\section{Christine Bagge et Alan Manning}

Volume 52, numéro 3, septembre 2007

URI : https://id.erudit.org/iderudit/016739ar

DOI : https://doi.org/10.7202/016739ar

Aller au sommaire du numéro

\section{Éditeur(s)}

Les Presses de l'Université de Montréal

ISSN

0026-0452 (imprimé)

1492-1421 (numérique)

Découvrir la revue

Citer cet article

Bagge, C. \& Manning, A. (2007). Grammar and Translation: The Noun + Noun Conundrum. Meta, 52(3), 556-567. https://doi.org/10.7202/016739ar

\section{Résumé de l'article}

Cet article aborde la question épineuse de la traduction en français des syntagmes nominaux anglais ayant pour structure Nom1 + Nom2. Prenant pour point de départ les quinze catégories sémantiques établies par Biber et al. (1999 : 589-591), revues et corrigées, les auteurs augmentent le nombre d'exemples afin d'en avoir vingt pour chaque catégorie et traduisent ces exemples en français. Ils montrent ensuite, à l'aide d'études de cas provenant de la traduction en français de plusieurs syntagmes nominaux anglais par des étudiants, que les étudiants dont la langue maternelle est l'anglais semblent avoir des difficultés à rendre le sens de certaines de ces structures et que, par contre, les étudiants faisant partie de l'échantillon dont la langue maternelle est le français ont tendance à commettre des erreurs que leurs homologues anglais ne font pas. Les implications pédagogiques de ce projet pilote sont indiquées, et une évolution linguistique quant à l'utilisation en français de syntagmes nominaux constitués de deux noms est mentionnée.
Ce document est protégé par la loi sur le droit d'auteur. L'utilisation des services d'Érudit (y compris la reproduction) est assujettie à sa politique d'utilisation que vous pouvez consulter en ligne.

https://apropos.erudit.org/fr/usagers/politique-dutilisation/ 


\title{
Grammar and Translation: The Noun + Noun Conundrum
}

\author{
CHRISTINE BAGGE \\ Coventry University, Coventry, United Kingdom \\ c.bagge@coventry.ac.uk
}

\author{
ALAN MANNING \\ Université Laval, Québec, Canada \\ alan.manning@lli.ulaval.ca
}

\begin{abstract}
RÉSUMÉ
Cet article aborde la question épineuse de la traduction en français des syntagmes nominaux anglais ayant pour structure Nom1 + Nom2. Prenant pour point de départ les quinze catégories sémantiques établies par Biber et al. (1999: 589-591), revues et corrigées, les auteurs augmentent le nombre d'exemples afin d'en avoir vingt pour chaque catégorie et traduisent ces exemples en français. Ils montrent ensuite, à l'aide d'études de cas provenant de la traduction en français de plusieurs syntagmes nominaux anglais par des étudiants, que les étudiants dont la langue maternelle est l'anglais semblent avoir des difficultés à rendre le sens de certaines de ces structures et que, par contre, les étudiants faisant partie de l'échantillon dont la langue maternelle est le français ont tendance à commettre des erreurs que leurs homologues anglais ne font pas. Les implications pédagogiques de ce projet pilote sont indiquées, et une évolution linguistique quant à l'utilisation en français de syntagmes nominaux constitués de deux noms est mentionnée.
\end{abstract}

\begin{abstract}
This article deals with the vexed question regarding the translation into French of English NOUN $1+$ NOUN2 sequences. Using the 15 meaning categories presented by Biber et al. (1999: 589-591), with some modifications and corrections, the authors expand each category into 20 representative items and translate them into French; they then show, by means of case study based on the translation into French of several noun sequences, that students whose first language is English seem to have difficulty rendering certain of these structures; by contrast, students participating in the study whose first language is French tend to commit errors not made by their English counterparts. The pedagogical implications of this pilot project are pointed up, and new linguistic developments concerning the use NOUN $1+N_{1} N_{2}$ in French are identified.
\end{abstract}

\section{MOTS-CLÉS/KEYWORDS}

noun + noun sequences, meaning transfer, case study, pedagogical implications, linguistic trends

\section{Introduction}

In our long years of teaching translation on both sides of the Atlantic, one of the most enduring grammatical problems for both native French-speakers and native English- 
speakers has focussed on noun + noun structures. This article addresses this issue primarily from an English-French point of view.

\section{Classifying Adjectives and the Noun + Noun Sequence}

Classifying adjectives identify nouns as being particular types. They fall into three groups, as follows:

1. adjectives proper (scientific, dental, agrarian),

2. -'s adjectives (a man's watch, a lady's bike, a butcher's knife) and

3. adjectival nouns (a coal mine, a glassworks, a river-bed, peace negotiations).

Noun + noun sequences belong to the latter category and are written as one word (glassworks), as two words joined by a hyphen (river-bed) or as two separate words (coal mine, peace negotiations). One-word and hyphenated adjectival nouns are generally lexicalized in most modern unilingual and bilingual dictionaries, including online dictionaries, and do not pose translation difficulties. On the other hand, adjectival noun structures are a highly productive and ongoing grammatical feature of modern English and are therefore not uniformly lexicalized. English grammarians have presented charts that categorize and explain such sequences but none, to our mind, as completely and coherently as Longman's Grammar of Spoken and Written English (Biber et al. 1999: 589-591). This chart will serve as the basis for our paper.

\section{The Structure and Meaning of Noun + Noun Sequences}

Noun + noun sequences contain only "content" words, with no function words to show the logical relations between the two parts. While they make it possible to squeeze a lot of information into few words, they are highly dependent on implicit meaning; that is, the reader has to infer the sense of the logical relationship underpinning sequences of adjectival and head nouns. Indeed, under Longman's classification, noun + noun groups fall into 15 basic semantic groups ( $\mathrm{N} 1 \Rightarrow$ adjectival noun, $\mathrm{N} 2 \Rightarrow$ head noun). We have categorized the list of sequences at the end of Longman's list and placed certain already classified items in the category that better corresponds to their meaning. We have highlighted these items with an asterisk. The modified list now reads as follows:

- Composition: $\mathrm{N} 2$ is made from N1, consists of N1, e.g. glass windows $\Rightarrow$ windows made from glass: word classes, protein granules, fact sheets, egg masses, metal seat, plastic beaker, zinc supplement, tomato sauce, satin dress, water supplies

- Purpose: N2 is for the purpose of N1; N2 is used for N1, e.g. pencil case $\Rightarrow$ used for pencils: safety device, search procedure, reference values, worship services, war fund, nursery program, extortion plan, chess board, radio station, brandy bottle, patrol car, Easter eggs, picnic ham, leg room, ${ }^{*}$ press release, ${ }^{*}$ computation times

- Identity: N2 has the same referent as N1 but classifies it in terms of different attributes, e.g. women algebraists $\Rightarrow$ algebraists who are women: conventionalism judge, men workers, consultant cardiologist, member country, exam papers, compression process, grant aid, ${ }^{*}$ jet streams, ${ }^{\star}$ bank holiday, ${ }^{\star}$ pressure hose, ${ }^{\star}$ pressure ratios, ${ }^{*}$ confidence trick, ${ }^{*}$ retail outlets

- Content: N2 is about N1; N2 deals with N1, e.g. algebra text $\Rightarrow$ a text about algebra; probability profile $\Rightarrow$ profile showing probability; currency crisis $\Rightarrow$ crisis relating to 
currency: sex magazines, market report, set theory, sports diary, prescription chart, success rates, credit agreement, intelligence bureau, explosives charges, interest group, speech impediment, colour adjective

- Source: N2 is from N1, e.g. irrigation water $\Rightarrow$ water that comes from irrigation: $c r o p$ yield, farmyard manure, plant residues, fault blocks, computer printout, Pentagon proposals, whale meat, ${ }^{*}$ media events, ${ }^{*}$ union assets

- Objective Type 1: N1 is the object of the process described in N2, or of the action performed by the ${ }^{*}$ inanimate agent described in $\mathrm{N} 2$, e.g. egg production $\Rightarrow \mathrm{X}$ produces eggs: waste disposal, paddy cultivation, root development, case study, water loss, ${ }^{*}$ child cruelty

- Objective Type 2: N2 is the object of the process described in N1, e.g. discharge water $\Rightarrow$ water that has been discharged: substitute forms, pilot projects

- Subjective Type 1: N1 is the subject of the process described in N2; N2 is a noun derived from an intransitive verb, e.g. child development $\Rightarrow$ children develop: leaf appearance, eye movement, management buy-out

- Subjective Type 2: N2 is the subject of the process described in N1, e.g. labour force $\Rightarrow$ a force that labours/is engaged in labour

- Time: N2 is found at the time given by N1, e.g. summer conditions, Sunday school, Christmas raffle

- Location Type 1: N2 is found or takes place at the location given by N1: corner cupboard, roof slates, Paris conference, home areas, world literature, church square, surface traction, tunnel trains, thigh injury, heart attack, industry sources

- Location Type 2: N1 is found at the location given by N2, e.g. notice board a board where notices are found: job centre, sushi bar, staff room, theme park

- Institution: N2 identifies an institution for N1, e.g. insurance companies $\Rightarrow$ companies for (selling) insurance: ski club, egg industry

- Partitive: N2 identifies parts of N1: cat legs, rifle butt, family member

- Specialization: N1 identifies an area of specialization for the person or occupation given in N2; N2 is animate, e.g. finance director $\Rightarrow$ director who specializes in finance: Education secretary, gossip columnists, football fans, estate agent, management consultant, ${ }^{*}$ taxi driver, ${ }^{*}$ administration officials, ${ }^{*}$ curio sellers, ${ }^{*}$ corn farmer, ${ }^{*}$ computer users

\section{Translating Noun + Noun Sequences into French: A Working Hypothesis}

Our aim in this article is to test the hypothesis that teaching students how to render English noun + noun sequences into French can be facilitated by presenting them with a system of equivalences based on the translation of each of the 15 semantic categories. As we did not have access to the Longman data base at the time of writing, we consulted a number of qualified informants to determine the effective meaning of each sequence. We translated the items in the chart into French, and in order to widen our scope of illustrations, we added sequences of our own (underlined), drawn from a wide variety of English specialized and semi-specialized sources. We also added in capitals the noun + noun sequences from "Cry Wolf?," a short passage that we translated into French and which we will use in the final part of this article in a case study of the contextual problems of translating noun + noun sequences. 
- Composition: glass windows /fenêtres, word classes/classes de mots, protein granules/ granules de protéines, fact sheets/fiches d'informations, egg masses/masses d'œufs or masses ovigères, metal seat/sièges métalliques, plastic beaker/gobelet en plastique, zinc supplement/supplément de or en zinc, tomato sauce/sauce tomate, satin dress/robe de satin, water supplies/approvisionnement en eau, terrorist networks/réseaux terroristes, paper trail/trace documentaire, pine grove/pinède, daisy chain/ guirlande or collier de pâquerettes, horror film/film d'épouvante or d'horreur, bone china/porcelaine, grass verge/bordure en gazon, iron will/volonté de fer, candy floss/barbe à papa

- Purpose: pencil case/trousse, safety device/dispositif de sécurité, search procedure/procedure d'enquête or de recherche, reference values/valeurs de référence, worship services/ offices religieux, war fund/souscription pour la guerre, nursery program/programme d'école maternelle, extortion plan/plan d'extorsion, chess board/échiquier, radio station/ station de radio or poste émetteur brandy bottle/bouteille de cognac, patrol car/voiture de police, Easter eggs/oeufs de Pâques, picnic ham/jambonneau, leg room/place pour les jambes, ${ }^{\star}$ press release/communiqué de presse, ${ }^{\star}$ computation times/temps utilisés dans les calculs, ${ }^{*}$ confidence trick/escroquerie, ${ }^{*}$ retail outlets/points de vente, tool box/boîte à outils

- Identity: women algebraists /des algébristes, conventionalism judge /juge conventionnaliste, men workers/ouvriers, consultant cardiologist/cardiologue, member country/état membre, exam papers/épreuves ou copies, compression process/procédé d'injection sous vide et pression, grant aid/subvention de l'Etat, ${ }^{*}$ voice communication/communication par la voix, ${ }^{\star}$ jet streams/courants-jets or jet-streams, ${ }^{\star}$ bank holiday/jour férié, ${ }^{\star}$ pressure hose/tuyau à pression, ${ }^{*}$ pressure ratios/rapports de pression, junk food/malbouffe, greenhouse gases/gaz contribuant à l'effet de serre, framework agreement/accord-cadre, war chest/caisse du parti or de grève, fairy godmother/fée marraine, noise pollution/nuisances sonores, cargo plane/avion-cargo.

- Content: algebra text /livre d'algèbre, probability profile /profil de probabilité, currency crisis /crise financière, sex magazines/revues pornographiques, market report/rapport de conjoncture, set theory/theories des ensembles, sports diary/journal sportif, prescription chart/feuille de prescription, success rates/taux de réussite, credit agreement/convention de crédit, intelligence bureau/service des renseignements, explosives charges/charges explosives, interest group/groupe d'intérêt(s), speech impediment/défaut d'élocution, color adjectives/adjectifs de couleur, road map/feuille de route, speed limit/vitesse limite, war clouds/signes avant-coureurs de la guerre, budget deficit/déficit budgétaire, personality disorder/trouble caractériel

- Source: irrigation water /eau d'irrigation, crop yield/rendement des cultures, farmyard manure/fumier, plant residues/déchets végétaux, fault blocks/blocs faillés, computer printout/imprimé d'ordinateur, Pentagon proposals/propositions du Pentagone, whale meat/ viande de baleine, court messengers/messagers ou envoyés de la cour, *union assets/ capital d'un syndicat, ${ }^{*}$ media events/événements médiatiques, cigarette butts/mégots, tap water/eau du robinet, tsunami victim/victimes du tsunami or des tsunamis, budget reaction/ réactions au budget, volcano lava/lave volcanique, crowd disturbances/troubles causés par la foule, chicken influenza/grippe aviaire, hate crimes/crimes inspirés par la haine, rust stain/tâche de rouille

- Objective Type 1: egg production/production d'œufs, waste disposal/évacuation des déchets, paddy cultivation/riziculture, root development/enracinement, case study/étude de cas, water loss/perte d'eau, child cruelty/cruauté envers les enfants, milk pasteurization/pasteurisation du lait, water purification/épuration de l'eau, food processor/robot ménager, traffic control/prévention routière, wind generator/éolienne, water heaterl chauffe-eau, plant closures/fermetures d'usine, road drill/marteau-piqueur, witness 
subpoena/citation d'un témoin, property fraud/ arnaque immobilière, time travel/les voyages dans le temps, coffee filter/filtre à café, graves desecration/profanation de tombes

- Objective Type 2: discharge water /eaux usées, substitute forms/succédanés, pilot projects/projets pilotes, advance notice/préavis, exchange student/étudiant d'échange, transfer credits/crédits or unités de valeur transférés dans le cadre d'une entente universitaire, reverse film/film inversible, graft tissue/tissu greffé, discount prices/prix d'escompte, retail items/articles vendus au détail, pull chord/tirette, torture victim/supplicié, download movie/film téléchargé, drill hole/perforation, contract work/travail à contrat, flood chamber/sas, throw switch/interrupteur, draw curtains/doubles rideaux, push button/poussoir, flood plain/plaine inondable

- Subjective Type 1: child development/développement de l'enfant, leaf appearance/ éclosion des feuilles, eye movement/mouvement oculaire, management buy-out/rachat d'une entreprise par ses cadres ou sa direction, pit explosion/coup de grisou, metal fatigue/fatigue du metal, climate change/changement climatique, gas explosion/explosion au gaz, population increase/augmentation démographique, isotope decay/désintégration isotopique, price escalation/escalade des prix, skin eruption/éruption cutanée, class struggle/lutte des classes, heart failure/arrêt cardiaque, court ruling/jugement rendu par la cour, oxygen depletion/épuisement d'oxygène, building collapse/effondrement d'un édifice, glacier retreat/recul des glaciers, WOLF ATTACK/ATTAQUE DE LOUPS, WOLF THREAT/MENACE QUE REPRÉSENTE LE LOUP

- Subjective Type 2: labour force/main d'œuvre or population active, con artist/arnaqueur, negotiation team/équipe de négociation, entertainment world/monde du spectacle, occupation force/force d'occupation, support wall/mur de soutènement, empowerment tool/outil d'autonomie, inflation trigger/déclencheur d'inflation, distribution network/ réseau de distribution, strike force/force de frappe, demolition squad/équipe de démolition, construction worker/ouvrier du bâtiment, research team/équipe de chercheurs, abolition law/loi abolitionniste, cancellation fee/taxe d'annulation, abortion pill/pilule abortive, promotion campaign/campagne publicitaire, adjournment debate/débat de clôture, balance weight/contrepoids, incentive bonus/prime d'encouragement

- Time: summer conditions/un temps d'été, Sunday school/école du dimanche, Christmas raffle/tombola de Noël, Saturday edition/édition du samedi, winter storm/tempête d'hiver, Renaissance poetry/poésie de la Renaissance, day surgery/chirurgie ambulatoire ou d'un jour, spring cleaning/grand nettoyage or nettoyage de printemps, night schooll cours du soir, afternoon tea/thé de cinq heures, autumn leaves/feuilles mortes ou d'automne, April Fool/poisson d'avril, evening dress/tenue de soirée, weekend excursion/ balade de week-end, morning-after pill/pilule du lendemain, Sunday paper/journal du dimanche, morning sickness/nausée du matin, night watch/veille de nuit, lunch break/ pause de midi, (medieval) mystery plays/mystères (du moyen-âge)

- Location Type 1: corner cupboard/placard d'angle, roof slates/tuiles d'ardoise, Paris conference/conférence de Paris, home areas/aires d'habitation, world literature/litterature mondiale, church square/place de l'église, surface traction/traction de surface, tunnel trains/trains souterrains, thigh injury/blessure à la cuisse, heart attack/crise cardiaque, industry sources/sources or origines industrielles, road rage/agressivité au volant, London apartment/appartement londonien, room temperature/température ambiante, toy storel magasin de jouets, Palestine conflict/conflit en Palestine, ceiling light/plafonnier, prison yard/cour or préau de prison, MERCANTOUR AREA/REEGION DU MERCANTOUR, FAIRY-TALE MENACE/INCARNATION DU DANGER DANS LES CONTES DE FÉES 
- Location Type 2: notice board/panneau d'affichage, job centre/bureau de placement, sushi table//table à sushis, staff room/ salle des professeurs, theme park/parc à thème, corner store/magasin de proximité, computer laboratory/laboratoire d'informatique, crisis centre/cellule de crise, lumber yard/dépôt de bois d'œuvre, burial plot/concession funéraire, temperature chart/feuille de température, grandpa gang/gang de pépés, host country/pays d'accueil, watch pocket/gousset, machine room/atelier d'usinage, slum district/quartier pauvre, instrument panel/tableau de bord, breakfast menu/menu du petit déjeûner, arrow pouch/carquois, salmon farm/ferme d'élevage de saumon

- Institution: insurance companies /compagnies d'assurance, ski club/club de ski, egg industry/industrie des oeufs, health authority/autorité sanitaire, play station/console de jeux vidéo, training camp/camp d'entraînement, wine region/region viticole, detoxification centre/centre de désintoxication, retirement home/maison de retraite, shopping mall/centre commercial or d'achats, parade ground/terrain de manoeuvres, game park/ réserve naturelle, insane asylum/asile d'aliénés, research institute/institut de recherches, employment agency/agence de placement, head office/siège social, isolation ward/salle des contagieux, stock exchange/bourse, convention centre/palais des congrès, university hospital/centre hospitalier universitaire

- Partitive: cat legs/pattes de chat, rifle butt/crosse de fusil, family member/membre de la famille, telephone cable/cable de téléphone, bicycle handlebars/guidon de vélo ou bicyclette, table top/plateau de table, garage doors/portes de garage, desk drawer/tiroir de bureau, railway station/gare airport lounge/salon d'aéroport, sea floor/fond de la mer, magazine cover/couverture de magazine, tent peg/piquet de tente, eye socket/orbite (de l'œil), banana skin/peau de banane, river channel/lit de rivière, alarm bell//sonnette d'alarme, clock face/cadran (d'horloge), piano pedals/pédalier (de piano), hockey stick/ bâton de hockey, window frame/châssis de fenêtre

- Specialization: finance director/chef des finances, directeur des finances: Education secretary/ministre de l'Éducation, gossip columnists/échotiers, football fans/amateurs du football, estate agent/agent immobilier, management consultant/conseiller en gestion, ${ }^{\star}$ taxi driver/chauffeur de taxi, ${ }^{*}$ administration officials/administrateurs, ${ }^{*}$ curio sellers/ brocanteurs, ${ }^{*}$ corn farmer/cultivateur de maïs, ${ }^{*}$ computer users/utilisateurs d'ordinateur, guidance counsellor/conseiller en orientation, business magnate/magnat de la finance, heart surgeon/chirurgien-cardiologue, football coach/entraîneur de football, book reviewer/critique littéraire, family therapist/thérapeute familial, sound engineer/ingénieur du son, SHEEP FARMER/ÉLEVEUR DE MOUTONS, ECOLOGY MINISTER/MINISTRE DE L'ÉCOLOGIE

\section{A Chart of French Equivalences of English Noun + Noun Sequences}

The following chart tabulates the 15 semantic noun + noun categories referred to above and lists the French structural translation patterns associated with each of them. For statistical purposes, when there were two equivalents given for a term in French, then the equivalent that was perceived to be the more common was used in the tables. The following caveat should be noted: If we assume that the 20 noun + noun sequences are typical of each category, then the different frequencies found should reflect the pattern of translations for each category. However, this can only be tested conclusively by taking a much larger random sample of noun + noun sequences from a large variety of sources. Similarly, noun + noun sequences are unlikely to be distributed evenly over the 15 categories and therefore the overall pattern of French translations could only be determined from a larger random sample. Our conclusions can therefore only be partial, however significant they may be. 


\begin{tabular}{|c|c|c|c|c|c|c|c|c|c|c|c|c|c|c|}
\hline $\mathrm{N} 1+\mathrm{N} 2$ & A & $\mathrm{B}$ & $\mathrm{C}$ & $\mathrm{D}$ & $\mathrm{E}$ & $\mathrm{F}$ & G & $\mathrm{H}$ & I & $\mathrm{J}$ & $\mathrm{K}$ & $\mathrm{L}$ & $\mathrm{M}$ & $\mathrm{N}$ \\
\hline $\begin{array}{r}1 \% \\
\%\end{array}$ & & $\begin{array}{c}7 \\
35\end{array}$ & $\begin{array}{c}4 \\
20\end{array}$ & $\begin{array}{c}4 \\
20\end{array}$ & $\begin{array}{c}3 \\
15\end{array}$ & $\begin{array}{l}1 \\
5\end{array}$ & $\begin{array}{l}1 \\
5\end{array}$ & & & & & & & \\
\hline $\begin{array}{r}2 \# \\
\%\end{array}$ & & $\begin{array}{l}11 \\
55\end{array}$ & & $\begin{array}{l}1 \\
5\end{array}$ & $\begin{array}{c}4 \\
20\end{array}$ & & & $\begin{array}{c}2 \\
10\end{array}$ & $\begin{array}{l}1 \\
5\end{array}$ & $\begin{array}{l}1 \\
5\end{array}$ & & & & \\
\hline $\begin{array}{r}3 \# \\
\%\end{array}$ & $\begin{array}{l}1 \\
5\end{array}$ & $\begin{array}{c}2 \\
10\end{array}$ & & $\begin{array}{c}4 \\
20\end{array}$ & $\begin{array}{c}5 \\
25\end{array}$ & $\begin{array}{l}1 \\
5\end{array}$ & & & $\begin{array}{c}2 \\
10\end{array}$ & $\begin{array}{l}1 \\
5\end{array}$ & $\begin{array}{l}1 \\
5\end{array}$ & $\begin{array}{c}3 \\
15\end{array}$ & & \\
\hline $\begin{array}{r}\# \\
\%\end{array}$ & $\begin{array}{c}2 \\
10\end{array}$ & $\begin{array}{l}10 \\
50\end{array}$ & & $\begin{array}{c}6 \\
30 \\
\end{array}$ & & $\begin{array}{l}1 \\
5 \\
\end{array}$ & & & $\begin{array}{l}1 \\
5 \\
\end{array}$ & & & & & \\
\hline $\begin{array}{r}5 \# \\
\% \\
\end{array}$ & $\begin{array}{c}5 \\
25 \\
\end{array}$ & \begin{tabular}{|c|}
4 \\
20 \\
\end{tabular} & & $\begin{array}{c}5 \\
25 \\
\end{array}$ & $\begin{array}{c}2 \\
10 \\
\end{array}$ & & & & $\begin{array}{c}2 \\
10 \\
\end{array}$ & & & & $\begin{array}{l}1 \\
5 \\
\end{array}$ & $\begin{array}{l}1 \\
5 \\
\end{array}$ \\
\hline $\begin{array}{r}6 \# \\
\% \\
\end{array}$ & $\begin{array}{c}3 \\
15 \\
\end{array}$ & $\begin{array}{c}5 \\
25 \\
\end{array}$ & & $\begin{array}{c}3 \\
15 \\
\end{array}$ & $\begin{array}{c}3 \\
15 \\
\end{array}$ & & & & $\begin{array}{c}2 \\
10 \\
\end{array}$ & $\begin{array}{l}1 \\
5 \\
\end{array}$ & & $\begin{array}{c}2 \\
10 \\
\end{array}$ & & $\begin{array}{l}1 \\
5\end{array}$ \\
\hline $\begin{array}{r}7 \# \\
\%\end{array}$ & & $\begin{array}{c}2 \\
10\end{array}$ & & $\begin{array}{c}5 \\
25\end{array}$ & $\begin{array}{c}8 \\
40\end{array}$ & $\begin{array}{l}1 \\
5\end{array}$ & $\begin{array}{l}1 \\
5\end{array}$ & & $\begin{array}{c}2 \\
10\end{array}$ & $\begin{array}{l}1 \\
5\end{array}$ & & & & \\
\hline $\begin{array}{r}8 \# \\
\% \\
\end{array}$ & $\begin{array}{c}5 \\
25 \\
\end{array}$ & $\begin{array}{c}4 \\
20 \\
\end{array}$ & & $\begin{array}{c}6 \\
30 \\
\end{array}$ & & & & & $\begin{array}{c}3 \\
15 \\
\end{array}$ & & & & $\begin{array}{l}1 \\
5 \\
\end{array}$ & $\begin{array}{l}1 \\
5 \\
\end{array}$ \\
\hline $\begin{array}{r}\% \# \\
\% \\
\end{array}$ & $\begin{array}{c}2 \\
10\end{array}$ & $\begin{array}{l}13 \\
65 \\
\end{array}$ & & $\begin{array}{c}3 \\
15 \\
\end{array}$ & $\begin{array}{c}2 \\
10 \\
\end{array}$ & & & & & & & & & \\
\hline $\begin{array}{r}10 \# \\
\%\end{array}$ & $\begin{array}{c}7 \\
35 \\
\end{array}$ & $\begin{array}{c}7 \\
35\end{array}$ & & $\begin{array}{c}3 \\
15\end{array}$ & $\begin{array}{l}1 \\
5\end{array}$ & & $\begin{array}{c}2 \\
10\end{array}$ & & & & & & & \\
\hline $\begin{array}{r}11 \# \\
\% \\
\end{array}$ & $\begin{array}{c}2 \\
10 \\
\end{array}$ & $\begin{array}{c}7 \\
35 \\
\end{array}$ & $\begin{array}{l}1 \\
5 \\
\end{array}$ & $\begin{array}{c}5 \\
25 \\
\end{array}$ & $\begin{array}{c}2 \\
10 \\
\end{array}$ & & & & $\begin{array}{l}1 \\
5 \\
\end{array}$ & & & & $\begin{array}{c}2 \\
10 \\
\end{array}$ & \\
\hline $\begin{array}{r}12 \# \\
\% \\
\end{array}$ & $\begin{array}{c}2 \\
10\end{array}$ & $\begin{array}{l}12 \\
60 \\
\end{array}$ & & $\begin{array}{c}2 \\
10 \\
\end{array}$ & $\begin{array}{c}2 \\
10 \\
\end{array}$ & & & & & $\begin{array}{c}2 \\
10 \\
\end{array}$ & & & & \\
\hline $\begin{array}{r}13 \# \\
\% \\
\end{array}$ & $\begin{array}{c}3 \\
15 \\
\end{array}$ & $\begin{array}{l}10 \\
50 \\
\end{array}$ & & $\begin{array}{c}6 \\
30 \\
\end{array}$ & $\begin{array}{l}1 \\
5 \\
\end{array}$ & & & & & & & & & \\
\hline $\begin{array}{r}14 \# \\
\% \\
\end{array}$ & $\begin{array}{c}2 \\
10 \\
\end{array}$ & $\begin{array}{l}15 \\
75 \\
\end{array}$ & & & $\begin{array}{c}3 \\
15 \\
\end{array}$ & & & & & & & & & \\
\hline $\begin{array}{r}15 \# \\
\% \\
\end{array}$ & $\begin{array}{c}5 \\
25 \\
\end{array}$ & $\begin{array}{c}6 \\
30 \\
\end{array}$ & $\begin{array}{c}2 \\
10 \\
\end{array}$ & $\begin{array}{c}3 \\
15 \\
\end{array}$ & $\begin{array}{c}3 \\
15 \\
\end{array}$ & & & & & & & $\begin{array}{l}1 \\
5 \\
\end{array}$ & & \\
\hline $\begin{array}{r}\text { Total \# } \\
\%\end{array}$ & $\begin{array}{l}39 \\
13 \\
\end{array}$ & $\begin{array}{c}115 \\
38.33 \\
\end{array}$ & $\begin{array}{c}7 \\
2.33 \\
\end{array}$ & $\begin{array}{c}56 \\
18,66 \\
\end{array}$ & $\begin{array}{l}39 \\
13 \\
\end{array}$ & $\begin{array}{c}4 \\
1.33 \\
\end{array}$ & $\begin{array}{c}4 \\
1.33 \\
\end{array}$ & $\begin{array}{c}2 \\
0.66\end{array}$ & $\begin{array}{c}14 \\
4.66 \\
\end{array}$ & $\begin{array}{l}6 \\
2 \\
\end{array}$ & $\begin{array}{c}1 \\
0.33 \\
\end{array}$ & $\begin{array}{l}6 \\
2 \\
\end{array}$ & $\begin{array}{c}4 \\
1.33 \\
\end{array}$ & $\begin{array}{l}3 \\
1\end{array}$ \\
\hline
\end{tabular}

Legend: (1) The structural pattern types of French translations:

$\mathrm{A}=\mathrm{N} 2+$ de + definite article $+\mathrm{N} 1$ (e.g. set theory/théorie des ensembles)

$\mathrm{B}=\mathrm{N} 2+\mathrm{de}+\mathrm{N} 1$ (e.g. algebra text/livre d'algèbre)

$\mathrm{C}=\mathrm{N} 2+$ en $+\mathrm{N} 1$ (e.g. plastic beaker/gobelet en plastique)

$\mathrm{D}=\mathrm{N} 2+$ adjective (e.g. sex magazines/revues pornographiques)

$\mathrm{E}=$ One word (e.g. picnic ham/jambonneau)

$\mathrm{F}=\mathrm{N} 2 \mathrm{~N} 1$ (e.g. member country/état membre)

$\mathrm{G}=$ idiom (e.g. candy floss/barbe à papa)

$\mathrm{H}=\mathrm{N} 2+$ pour + definite article $+\mathrm{N} 1$ (e.g. war fund/souscription pour la guerre)

$\mathrm{I}=$ periphrase (e.g. computation times/temps utilisés dans les calculs)

$\mathrm{J}=\mathrm{N} 2+\mathrm{a}+\mathrm{N} 1$ (e.g. tool box/boîte à outils)

$\mathrm{K}=\mathrm{N} 2+$ par+definite article $+\mathrm{N} 1$ (e.g. voice communication/communication par la voix)

$\mathrm{L}=\mathrm{N} 2-\mathrm{N} 1$ (e.g. cargo plane/avion-cargo)

$\mathrm{M}=\mathrm{N} 2+\mathrm{a}+$ definite article $+\mathrm{N} 1$ (e.g. budget reaction/réaction au budget)

$\mathrm{N}=\mathrm{N} 2+$ de+indefinite article $+\mathrm{N} 1$ (e.g. building collapse/effondrement d'un edifice)

Legend (2) The types of N1 + N2 categories:

$1=$ Composition

$2=$ Purpose

$3=$ Identity

$4=$ Content

$5=$ Source

6 = Objective Type 1

7 = Objective Type 2

$8=$ Subjective Type 1

$9=$ Subjective Type 2

$10=$ Time

11 = Location Type 1

$12=$ Location Type 2

$13=$ Institution

$14=$ Partitive

$15=$ Specialization 
The chart confirmed our initial general hypothesis that French pattern B $(\mathrm{N} 2+\mathrm{N} 1)$ is the most commonly adopted structure for translating all 15 English noun + noun sequences, followed, a long way behind, by patterns D (N2 + adjective) and, ex-aequo, $\mathrm{A}(\mathrm{N} 2+$ de + definite article $+\mathrm{N} 1)$ and $\mathrm{E}$ (one word). The popularity of the latter pattern came as a particular surprise to us. Our second surprise was to find that certain categories tend to cluster and predominate in specific semantic categories. For example: in category 3 (Identity), patterns E (one word) and D (N2 + adjective) are more prominent than category B; in category 5 (Source), patterns A $(\mathrm{N} 2+\mathrm{de}+$ definite article $+\mathrm{N} 1$ ) and D are more significant; in category 7 (Objective Type 2), pattern $\mathrm{E}$ (one word) is the most prominent structure; in category 8 (Subjective Type 1), patterns D and A are preferred; in category 10 (Time), patterns A and B are equally distributed; finally, in category 15 (Specialization), pattern B is just slightly ahead of pattern A. The pedagogical ramifications of these observations is obvious; namely that although pattern B is by far and away the most common French structural equivalence of English noun + noun sequences in general, its actual rate of incidence depends on the particular semantic grouping to which it belongs. That said, any equivalence chart will always be left wanting, since the act of translating does not involve transcoding or the simple transfer of one word or group of words from one language to another: style and context are equally decisive. In order to demonstrate the truth of this assertion, we analyzed student translations of an English magazine article that contained several noun + noun sequences. We subsequently added these sequences to our equivalence chart.

\section{Noun + Noun Sequences: A Case Study}

We selected an article from the magazine The Economist that had been translated into French by 15 students, all in their last year in the French Honours group at Coventry University, in the United Kingdom. The article - Crying wolf? - contained six noun + noun structures. One structure - sheep farmers - was unusually hyphenated in the original; after checking a number of informants as well as several websites, we restored its normal orthography. Seven of the group were native French-speakers and the remaining eight, native English-speakers. Though the former did not play a primary role in this study, we nonetheless retained their answers to see whether, like their English-speaking peers, they took account of style and context in their translations. Before comparing and contrasting the students' work, we did our own translation and assigned the noun + noun sequences to their individual patterns and categories. Thus:

\section{Crying wolf?}

Fairy-tale menace and biblical demon, the wolf is creeping into French politics. After a string of wolf attacks on sheep in the Southern Alps, Serge Lepeltier, the ecology minister, agreed to a cull. But the decision has prompted furious debate between pastoralists and ecologists, divided the government, and re-opened the whole question of what the French countryside is for.

At the start of the $19^{\text {th }}$ century, France had some 5,000 wolves. Then the wolf disappeared in the 1930s, chased by gunshot, poison and urbanisation. In 1992 wolves from Italy reappeared in the Southern Alps. Last year there were 508 wolf attacks, eight times as many as ten years ago, involving the loss of 2,177 sheep. Most took place in the Mercantour area of the Southern Alps. 
For their part, ecologists are livid about the cull. Olivier Rousseau, head of the Association for the Protection of Wild Animals, argues that sheep farmers should take advantage of the return of this majestic creature, and diversify into such activities as eco-tourism.

This touches the politically sensitive matter of how traditional French farming is to survive. Ecologists claim that farmers are exaggerating the wolf threat to cling to an outdated system of subsidies. Farmers retort that their business is production, not park keeping. "We will not tolerate Disneyfication," sniffs one sheep farmer.

The Economist, September $18^{\text {th }} 2004$

Suggested translation:

\section{Crier au loup?}

Incarnation du danger dans les contes de fées et du démon dans la Bible, le loup s'insinue dans la vie politique française. Après une série d'attaques de loups sur des moutons dans les Alpes du Sud, Serge Lepeltier, ministre de l'Ecologie, a autorisé un abattage sélectif. Or, cette décision a suscité une vive polémique entre pastoralistes et écologistes, divisé le gouvernement et rouvert le grand débat sur la vocation des campagnes françaises.

Au début du XIX ${ }^{e}$ siècle, la France comptait quelque 5000 loups. Par la suite, dans les années 1930, le loup a disparu, pourchassé par le tir, les empoisonnements ou l'urbanisation. En 1992, des loups venus d'Italie ont réapparu dans les Alpes du Sud. L'année dernière, on a dénombré 508 attaques de loups, soit huit fois plus qu'il y a dix ans, qui se sont soldées par la perte de 2177 moutons. La plupart se sont produites dans la région du Mercantour dans les Alpes du Sud.

Les écologistes, quant à eux, sont furieux qu'on ait autorisé l'abattage. Olivier Rousseau, président de l'Association pour la protection des animaux sauvages, soutient que les éleveurs de moutons devraient profiter du retour de ce majestueux animal pour diversifier leurs activités en se tournant notamment vers l'écotourisme.

Cette controverse soulève la question, sensible d'un point de vue politique, de la manière dont on envisage la survie de l'agriculture traditionnelle en France. Les écologistes prétendent que les éleveurs exagèrent l'importance de la menace que représente le loup pour conserver un système d'indemnisations dépassé. Les éleveurs, eux, rétorquent que leur rôle consiste à générer des biens agricoles et non à garder des parcs. "Nous ne tolérerons pas la Dysneyfication de nos campagnes», déclare avec dédain un éleveur de moutons.

Fairy-tale menace: (Incarnation du) danger dans les contes de fées. Location Type 1/ Pattern I

wolf attacks ( 2 occurrences): attaques de loups. Subjective Type 1/Pattern B

ecology minister: ministre de l'Ecologie. Specialization/Pattern A

Mercantour area: région du Mercantour. Location Type 1/Pattern A

wolf threat: menace que représente le loup. Subjective Type 1/Pattern I

sheep farmer: éleveur de moutons. Specialization/Pattern B

All 6 noun + noun sequences fit neatly into 3 semantic categories (Location Type 1, Subjective Type 1 and Specialization); they are translated by the forms predicted by our chart; two are translated using the pattern A (ecology minister, Mercantour area); two the pattern B (wolf attacks, sheep farmer); the remaining two (Fairy-tale menace, wolf threat) are expressed by the pattern I, phraseological in both cases.

Formally, Fairy-tale menace and Mercantour area belong to the same semantic category; however, the underlying meaning between the nouns in each sequence is not the same. In the first case, the author evokes a menace typically found in fairy 
tales, the meaning expressed in French by the "danger (qui se trouve) dans les contes de fées." In the second, the nouns are perceived as equivalent; in other words, Mercantour is an area, hence "région du Mercantour." If wolf threat were to be rendered by menace de loup, it would designate a type of threat. The sense is much more concrete, as the wolf is the agent of the action evoked in the head noun "threat," hence, the more explicit phrase "menace que représente le loup."

For reasons of style and lexis, we have included the following items in our discussion: biblical demon: démon dans la Bible; farmers: éleveurs. As with the latter example, we chose the symmetrical rendering démon dans la Bible because we considered it stylistically more appropriate than its variant demon biblique. In the second example, we considered it as an abbreviation of sheep farmers in this context and translated it in French by the abbreviation of éleveurs de moutons - éleveurs.

Next, we compiled an analytical chart of the students' translations of the noun + noun sequences. As mentioned earlier, we have recorded the answers of the 7 native French-speakers alongside their 8 English counterparts, in order to measure their sensitivity to contextual meaning.

\section{Analytical Chart of Students Noun + Noun Translations}

\begin{tabular}{|c|c|c|c|}
\hline TERMS & ENGLISH STUDENTS & FRENCH STUDENTS & OUR TRANSLATION \\
\hline fairy-tale menace & $\begin{array}{l}\text { comme la menace dans les } \\
\text { contes de fées } \\
\text { une menace de contes de fées } \\
\text { la menace des contes de fées } \\
\text { une menace de contes de fées } \\
\text { le menace de contes de fées } \\
\text { menace de contes de fées } \\
\text { une menace conte de fées } \\
\text { la menace enchanteresse }\end{array}$ & $\begin{array}{l}\text { menace de contes de fées } \\
\text { conte de fée qui se fait } \\
\text { menaçant } \\
\text { menace de contes de fées } \\
\text { symbole d'une menace dans } \\
\text { les contes de fées } \\
\text { menace enchanteresse } \\
\text { menace de contes de fées } \\
\text { menace des contes de fées }\end{array}$ & $\begin{array}{l}\text { Incarnation du danger } \\
\text { dans les contes de fées }\end{array}$ \\
\hline Wolf attacks & $\begin{array}{l}\text { attentats contre des } \\
\text { moutons } \\
\text { attaques de loup } \\
\text { attaques de loup } \\
\text { attaques de loup } \\
\text { attaques par les loups } \\
\text { attaques } \\
\text { loupes attaques } \\
\text { attaques du loup }\end{array}$ & $\begin{array}{l}\text { attaques de loups } \\
\text {...attaqués par des loups } \\
\text { attaques par les loups } \\
\text { attaques } \\
\text { attaques de loups } \\
\text { attaques de loups } \\
\text { attaques de loup }\end{array}$ & attaques de loups \\
\hline ecology minister & $\begin{array}{l}\text { ministre de l'Écologie } \\
\text { Ministre de l'Écologie } \\
\text { ministre de l'Écologie } \\
\text { ministère de l'écologie } \\
\text { ministre de l'écologie } \\
\text { ministre de l'écologie } \\
\text { ministre d'écologie } \\
\text { ministre d'écologie }\end{array}$ & $\begin{array}{l}\text { ministre de l'Écologie } \\
\text { Ministre de l'écologie } \\
\text { ministre de } \\
\text { l'écologie } \\
\text { ministre de l'Écologie } \\
\text { ministre de } \\
\text { l'environnement } \\
\text { ministre de } \\
\text { l'écologie } \\
\text { ministre de l'Écologie }\end{array}$ & ministre de l'Écologie \\
\hline Mercantour area & $\begin{array}{l}\text { région de Mercantour } \\
\text { région de Mercantour } \\
\text { région de Mercantour } \\
\text { région de Mercantour } \\
\text { région de Mercantour } \\
\text { région Mercantour } \\
\text { Mercantour région } \\
\text { région Mercantour }\end{array}$ & $\begin{array}{l}\text { région du Mercantour } \\
\text { région du Mercantour } \\
\text { région du Mercantour } \\
\text { zone du Mercantour } \\
\text { région du Mercantour } \\
\text { zone du Mercantour } \\
\text { région du Mercantour }\end{array}$ & région du Mercantour \\
\hline
\end{tabular}




\begin{tabular}{|c|c|c|c|}
\hline sheep farmers & $\begin{array}{l}\text { éleveurs de moutons } \\
\text { agriculteurs } \\
\text { éleveurs de moutons } \\
\text { éleveurs de mouton } \\
\text { pâturages à moutons } \\
\text { agriculteurs du mouton } \\
\text { agriculteurs } \\
\text { agriculteurs du mouton }\end{array}$ & $\begin{array}{l}\text { bergers } \\
\text { éleveurs de moutons } \\
\text { éleveurs de moutons } \\
\text { éleveurs de moutons } \\
\text { éleveurs de moutons } \\
\text { éleveurs de moutons } \\
\text { éleveurs de moutons }\end{array}$ & éleveurs de moutons \\
\hline wolf threat & $\begin{array}{l}\text { la menace du loup } \\
\text { la menace de loup } \\
\text { la menace de loup } \\
\text { la menace du loup } \\
\text { la menace du loup } \\
\text { l'histoire du loup } \\
\text { la menace de loupes } \\
\text { l'histoire du loup }\end{array}$ & $\begin{array}{l}\text { la menace du loup } \\
\text { la menace du loup } \\
\text { la menace que représente } \\
\text { le loup } \\
\text { la menace du loup } \\
\text { la menace du loup } \\
\text { la menace possible du loup } \\
\text { la menace du loup }\end{array}$ & $\begin{array}{l}\text { menace que représente } \\
\text { le loup }\end{array}$ \\
\hline
\end{tabular}

The scores of the English-speaking students were as follows:

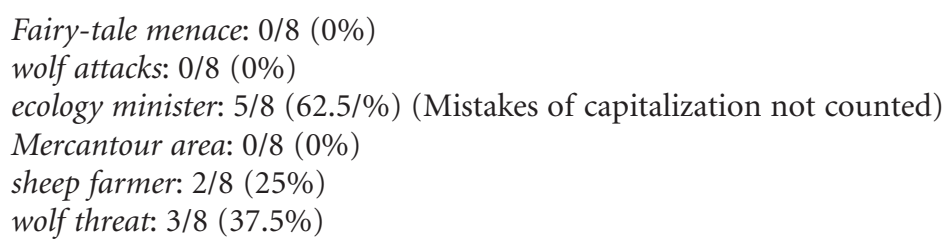

All the errors appear to arise from insufficient training in rendering noun + noun structures into French. In particular, the first sequence in the text, Fairy-tale menace, required that its contextual meaning be clearly distinguished from that of Mercantour area, that the contextual meaning of wolf threat be clearly ascertained, and that stylistic adjustment in the interest of symmetry (biblical demon) be made, as we mentioned earlier.

The native French-speaking students scored as follows:

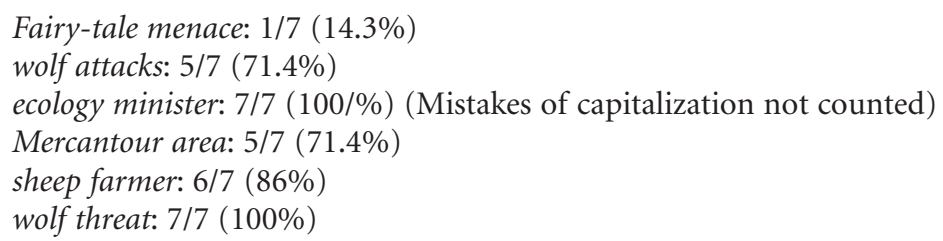

They were patently the stronger of the two groups, given their linguistic advantage; however, they were likewise found wanting in the following categories: Location Type 1 (Fairy-tale menace), and Subjective Type 1 (wolf threat). In other words, both English and French lacked sensitivity to contextual meaning and stylistic variation.

\section{Conclusion}

There is no doubt in our minds that, based on the results of our Case Study, many of the students who took part in it would benefit from exposure to the equivalence chart that we have presented in this article. Although the chart provides many of the solutions related to the meaning and translation of noun + noun sequences, our case study underlines the rule that the language student and the future translator should 
be constantly aware of contextual meaning and individual stylistic requirements. After all, is not the primary goal of language transfer to convey the message, not the form? Students commit errors because they do the opposite, as we have seen in the tabulation of their errors. In particular, the noun + noun sequence will cease to be a conundrum for them once they have grasped and begun applying this rule, along the lines that we have laid down for them in this article.

The difficulty of translating the noun + noun sequences into French naturally stems from the fact that this structural pattern is not characteristic of French. As we mentioned earlier, noun + noun sequences make it possible to squeeze a lot of information into few words and accelerate the communication process. This may be the reason why they seem gradually to be finding their way into the French language through catchy political and journalistic phrases. La positive attitude of Former Prime Minister Jean-Pierre Raffarin (See the editorial by M.Ulrich in Libération of 21 January 2005) apparently set the stage when he used the phrase la positive attitude, a patently English structure putting the adjective before the noun. It seemed to give respectability to this pattern, at least with regard to the word attitude. For example, in its 28 May 2005 issue, Le Figaro contains the phrase La fraîche attitude to describe La semaine de découverte et de dégustation des fruits de mer et des légumes frais; the 11 July 2005 issue of L'Express contains an article on La blog attitude, a clearly English noun + noun structure. Will this system of classifying nouns into particular types become widespread in French? If ever it does happen, the process will occur over a very long period of time. In the meantime, it does not undermine the validity of our findings.

\section{REFERENCES}

Attal, J.-P., Tothill, G. S. et A. Swan (1987): Grammaire et usage de l'anglais, Paris-Gembloux, Duculot.

Biber, D., Johansson, S., Leach, G., Conrad, S. and E. Finegan (1999): Grammar of Spoken and Written English, Harlow, Longman.

Chuquet, H. et M. Paillard (1989): Approche linguistique des problèmes de traduction, Paris, Ophrys.

DéLÉPINe, S. (2003): La grammaire anglaise de l'étudiant, Paris, Ophrys.

Grellet, S. (1993): Initiation au thème anglais: The Mirrored Image, Paris, Hachette.

Huddlestone, R. and G. K. Pullum (2002): The Cambridge Grammar of the English Language, Cambridge, Cambridge University Press.

Malavielle, J. et W. Rotgé (1997): La grammaire anglaise, Paris, Hatier.

Marcelin, J., Faivre, F., Garner, C., Ratié, M. (1996): Grammaire de l'anglais, Paris, Hatier.

Swan, M. (2005): Practical English Usage, Oxford, Oxford University Press. 